and $52 \mathrm{~d}$ ) during food re-introduction (FR). Microbiome was assessed by $16 \mathrm{~s}$ rRNA gene sequencing of the V4 region performed on the MiSeq (Illumina). Community structure was resolved at $97 \%$ similarity operational taxonomic unit (OTU). Short chain fatty acids (SCFA) were quantified with gas chromatography and are expressed in $\mu \mathrm{mol} / \mathrm{g}$. Faecal calprotectin (FC) was measured using the CALPROLAB0170 (ALP) (Lysaker, Norway) ELISA kit. Continuous data are present as mean and standard deviation unless otherwise stated.

Results 66 CD patients were recruited (Female 25; age 13.4 yr). Clinical remission (wPCDAI<12.5) was achieved in 41 (62\%). During EEN there was an increase in Shannon diversity (start: 0.3 [0.22] vs 30d EEN: 0.48 [0.2], p<0.001; vs 56d EEN: 0.43 [0.27], $\mathrm{p}=0.05)$. During FR these indices did not change.

Based on $\beta$-diversity dispersion analysis, estimated using Bray-Curtis distance, EEN induced clear alterations to the microbiome. Permutation ANOVA was used to identify significant changes to the microbiome during EEN. Most of the change that occurred was apparent within the first 4 weeks of treatment with R2: 4.7\%, $(\mathrm{p}=0.001)$ and by the end of EEN R2: $3.2 \%,(p=0.001)$.

In patients to enter remission using EEN, we observed a quick reversion in the microbiome composition to that of pretreatment $(\mathrm{p}=0.23)$.

Assessing the metabolic activity of the microbiome we observed a significant decrease in the concentration of acetate (start: 423.6 [183.6], end: 224.9 [101.5]; $\mathrm{p}<0.001$ ), propionate (start: 93.8 [50.6], end: 55.7 [27.3]; p< 0.001) and butyrate (start: 95.0 [64.2], end: 41.0 [50.7]; $\mathrm{p}<0.001$ ). During FR, there was a rapid reversion in levels of acetate and propionate (acetate EEN end: 224.9 [101.5] vs 17d FR: 362.4 [179.7]; $p=0.003$; propionate EEN end: 55.7 [27.3] vs 17d FR: 93.0 [46.9]; $\mathrm{p}=0.002$ ).

Faecal calprotectin significantly decreased during EEN (start: 1402.4 [586.3]; 4wk EEN: 877.5 [593.1], p <0.001; $8 w k$ EEN: 720 [664], $\mathrm{p}<0.001)$ and was quickly reversed during food re-introduction (17d FR: 1025 [603], $\mathrm{p}=0.025$; 52d FR: 1105 [651], p=0.003)

Conclusions EEN induces specific effects on faecal microbiome and markers of functional activity. This is characterised by a reduction in metabolic activity during EEN, with reversion to pre-EEN state during food re-introduction paralleling an elevation of faecal calprotectin

\section{REGULATION AND ROLE OF ALPHAE INTEGRIN IN MIGRATION AND RETENTION OF LYMPHOCYTES IN INTESTINAL MUCOSA}

\footnotetext{
1,2Christopher A Lamb*, ${ }^{3}$ Bingbing Dai, ${ }^{3}$ Ryan Ichikawa, 1,2 Meghan Acres, ${ }^{1}$ Gillian Hulme, ${ }^{1,2}$ Christopher D Carey, ${ }^{1}$ Jeremy Palmer, ${ }^{2}$ Claire J Jones, ${ }^{2}$ Anna K Long, ${ }^{1,2}$ John C Mansfield, ${ }^{3}$ Justin Elstrott, ${ }^{3}$ Alvin Gogineni, ${ }^{1}$ Andrew Filby, ${ }^{1}$ John A Kirby, ${ }^{3}$ Jacqueline McBride, ${ }^{3}$ Mary E Keir, ${ }^{3}$ Tangsheng Yi. ${ }^{1}$ Newcastle University, Newcastle Upon Tyne, UK; ${ }^{2}$ Newcastle upon Tyne Hospitals NHS Foundation Trust, Newcastle upon Tyne, UK; ${ }^{3}$ Genentech Research and Early Development, South San Francisco, USA
}

\subsection{6/gutjnl-2020-bsgcampus.13}

Introduction Targeting integrins that mediate adhesion and migration of lymphocytes to the gastrointestinal (GI) tract is an effective therapy in inflammatory bowel disease (IBD). $\alpha 4 \beta 7$ and $\alpha 4 \beta 1$ are expressed on circulating lymphocytes that may mediate inflammation, while $\alpha E \beta 7$ integrin is expressed primarily on a subset of $\mathrm{T}$ cells within the mucosa.
Etrolizumab is a humanized monoclonal antibody that selectively binds the $\beta 7$ subunit of the $\alpha 4 \beta 7$ and $\alpha \mathrm{E} \beta 7$ integrin heterodimers. The relative role of individual integrin heterodimers in lymphocyte migration and retention in the GI tract remains to be characterized.

Methods pSMAD3, MAdCAM, VCAM and ICAM levels were measured in colonic and ileal biopsies. $\alpha 4 \beta 7+$ and $\alpha 4 \beta 7-$ human $\mathrm{T}$ cells were induced to express $\alpha \mathrm{E}$ integrin by TGF- $\beta$ 1 stimulation followed by qPCR array gene expression analysis. A murine photo-convertible reporter system was used to determine the effect of blockade of $\alpha 4 \beta 7$ and/or $\alpha E \beta 7$ integrins on lymphocyte migration and retention. $\mathrm{T}$ cell-epithelial cell interactions were evaluated using intravital two-photon microscopy.

Results pSMAD3 was observed in the epithelium and lamina propria in IBD biopsies, suggesting active TGF- $\beta$ signalling. Adhesion molecule expression was increased in inflamed biopsies. TGF- $\beta 1$ stimulation induced $\alpha \mathrm{E}$ integrin expression on both $\alpha 4 \beta 7+$ and $\alpha 4 \beta 7$ - circulating $\mathrm{T}$ cells. $\alpha \mathrm{E} \beta 7+$ cells derived from $\alpha 4 \beta 7+$ and $\alpha 4 \beta 7$ - progenitors had similar cytokine, chemokine, transcription factors and effector molecule gene expression. In a mouse model of $\mathrm{T}$ cell migration, combined blockade of both $\alpha 4 \beta 7$ and $\alpha \mathrm{E} \beta 7$ with anti- $\beta 7$ (etrolizumab surrogate) led to a greater reduction of $\mathrm{T}$ cell accumulation in the intestinal mucosa and epithelium compared to single blockade of either $\alpha 4 \beta 7$ or $\alpha E \beta 7$. Further intravital two-photon microscopy and photo-specific labelling experiments revealed that blockade of $\alpha E \beta 7$ reduces $T$ cell:epithelial cell interactions, increases the migratory speed of activated $T$ cells in the intestinal mucosa, and facilitates effector $\mathrm{T}$ cell egress from the intestinal mucosa through lymphatic vessels.

Conclusions $\alpha E \beta 7$ is induced by TGF- $\beta 1$ on both $\alpha 4 \beta 7+$ or $\alpha 4 \beta 7$ - T cells. Co-blockade of $\alpha 4 \beta 7$ and $\alpha E \beta 7$ together leads to greater inhibition of $\mathrm{T}$ cell accumulation in gastrointestinal tissues through a stepwise inhibition of $\mathrm{T}$ cell migration and subsequent tissue retention.

\section{WHOLE BLOOD PROFILING OF T-CELL DERIVED MIRNA ALLOWS THE DEVELOPMENT OF PROGNOSTIC MODELS IN IBD}

${ }^{1}$ Rahul Kalla*, ${ }^{2}$ Alex Adams, ${ }^{3}$ Ruby White, ${ }^{4}$ Carolyn Clarke, ${ }^{3}$ Alasdair Ivens, ${ }^{5}$ Nicholas Ventham, ${ }^{6}$ Nicholas Kennedy, ${ }^{7}$ Seanna McTaggart, ${ }^{1}$ IBD Character Consortium, ${ }^{1}$ Gwo-Tzer Ho, ${ }^{3}$ Amy Buck, ${ }^{2} J a c k$ Satsangi. 'MRC Centre for Inflammation Research, University Of Edinburgh, Edinburgh, UK; ${ }^{2}$ Nuffield Department of Medicine, University of Oxford, Oxford, UK; ${ }^{3}$ Institute of Immunology and Infection Research and Centre for Immunity, Infection and Evolution, School of Biological Sciences, University of Edinburgh, Edinburgh, UK; ${ }^{4}$ LifeArc, Edinburgh, UK; ${ }^{5}$ Centre for Genomic and Experimental Medicine, Edinburgh, UK; ${ }^{6}$ Exeter IBD and Pharmacogenetics group, University of Exeter, Exeter, UKi ${ }^{7}$ Earlham Institute, Norwich, UK

\subsection{6/gutjnl-2020-bsgcampus. 14}

Introduction There is an unmet need for blood-based biomarkers that help predict disease and its course at inception to allow tailoring of treatments, achieve early mucosal healing and improve clinical outcomes. In our study, we explore the clinical utility of miRNAs in Inflammatory bowel disease (IBD).

Methods A 2-stage prospective multi-centre case control study was performed. Small RNA sequencing was performed on a discovery cohort of immunomagnetically separated leucocytes (90 CD4+ \& CD8+ T-lymphocytes and CD14+ monocytes) 